\title{
Coronary artery fistulae and treatment in children
}

\author{
Kahraman Yakut $^{1 \oplus}$, N. Kürşad Tokel ${ }^{1 \oplus}$, Birgül Varan ${ }^{1 \oplus}$, İlkay Erdoğan $^{1 \oplus}$, \\ Murat Özkan ${ }^{2 \odot}$ \\ Departments of ${ }^{1}$ Pediatric Cardiology and ${ }^{2}$ Cardiovascular Surgery and Başkent University Faculty of Medicine, Ankara, Turkey.
}

\begin{abstract}
Background and objectives. In this study, we aimed to review the treatment options and long-term problems of patients who were diagnosed with coronary artery fistulae (CAF) in our institution. We also tried to determine the most appropriate time for treatment of this condition.
\end{abstract}

Method. From 2000 to 2018, the medical records of 56 patients (33 males and 23 females) who had CAF diagnoses were retrospectively reviewed.

Results. The mean age of the patients at the time of diagnosis was $3.9 \pm 4.6$ years (range, 1 month to 18 years) and the mean duration of the follow-up period was $7.4 \pm 4.5$ years (range, 1 year to 17.5 years).

The right coronary artery (RCA) was the most common origin site for CAF, the left main coronary artery (LMCA) was the second most common origin site whereas the left anterior descending coronary artery (LAD) was the third most common origin site. Catheter angiography showed that right ventricle (RV) was the site of termination for CAF in 23 patients $(41.1 \%)$ while the CAF drained to the pulmonary artery in 16 patients (28.6\%). Transcatheter intervention was performed in ten patients, while CAF were corrected surgically in five patients. Transcatheter intervention was initially attempted in two out of the five surgically-treated patients, but the procedure was unsuccessful. A vascular plug was deployed in six patients, a platinum coil was used in three patients, and a platinum coil with tissue adhesive was placed in one patient using a catheter. Early complications were seen in two patients during transcatheter intervention and in one patient during surgery. There were no instances of death or late complications in patients treated surgically or via transcatheter.

Conclusions. Coronary artery fistulae are usually asymptomatic, and medical therapy with long term follow up is the first line treatment. Fistulae that cause hemodynamically significant shunting, chamber enlargement, or visible symptoms should be closed at an early age. This study shows that transcatheter closure is a safe treatment option for CAF that may be performed with high success. Also, it should be known that surgery may be performed effectively with low rates of complications. Because complications can develop in treated and untreated patients of all ages, follow-up should occur during the patient's lifetime.

Key words: coronary artery fistulae, angiography, transcatheter closure, surgery treatment.

Coronary artery fistulae are rarely seen, and they are usually asymptomatic. ${ }^{1-5}$ The clinical relevance of CAF focuses mainly on the mechanism of 'coronary steal phenomenon', which causes myocardial functional ischaemia even in the absence of stenosis. Common symptoms of this phenomenon are angina,

¿ahraman Yakut

kahramanyakut@gmail.com

Received 10th August 2019, revised 20th October 2019, 16th February 2020, accepted 30th March 2020. dyspnoea during physical exertion, and heart palpitations. ${ }^{2-5}$ The suggested diagnostic approach is guided by the patient's symptoms and consists of a number of instrumental examinations such as the electrocardiogram, treadmill test, echocardiography, computed tomography and coronary angiography. Bacterial endocarditis is rarely observed in patients with CAF.6,7 Small, asymptomatic fistulae can be followed by echocardiography, while treatment of symptomatic or large asymptomatic fistulae is recommended..$^{2-5,8,9}$ Coronary angiography is required to plan 
optimal management of CAF. Invasive treatments, such as transcatheter or surgical approaches, are usually a reasonable choice for selected patients. The long-term results of these strategies are similar. Transcatheter closure is the treatment of choice of our clinic; however, surgery is performed in patients with fistulae when transcatheter closure is not feasible. Both treated and untreated patients should have a lifelong follow-up period.

\section{Material and Methods}

The demographic features, clinical findings, electrocardiographic, echocardiographic and angiographic data from 56 patients (33 males, 23 females) with CAF were reviewed, and the clinical courses of treated patients were examined. The approval for the study was obtained from our university ethics committee (KA18/208-26.06.2018). Associated lesions with CAF were described. Patients who had CAF associated with abnormalities related to the origin of the coronary artery, right ventricledependent coronary artery circulation, complex congenital heart defects (CHD) and patients with acquired CAF were excluded from this study. Coronary artery dilation, coronary steal syndrome, cardiac chamber enlargement, an increase in the ratio of shunt-induced flow (Qp/Qs>1.5), left ventricle hypertrophy and congestive heart failure were major treatment indications. Patients were followed regularly, and their condition was evaluated using electrocardiography and echocardiography. Two-dimensional (2D) and colour doppler images were obtained from parasternal long and short axis, apical four chamber, subcostal and suprasternal views with variable frequency transducers using the Vivid ${ }^{\mathrm{TM}} \mathrm{q}$ cardiovascular ultrasound system (GE Healthcare 9900 Innovation Drive Wauwatosa, WI 53226 USA). The coronary artery was considered to be dilated when its diameter was measured by using echocardiography higher than +2 standard deviation (SD). The Artis zee biplane system (Siemens Healthcare Headquarters Siemens Healthcare GmbH Henkestr. 127
91052 Erlangen Germany) was used for catheter angiography.

\section{Statistical analysis}

Statistical analysis was performed using the SPSS software version 21 (IBM Analytics, Armonk, New York, USA). Descriptive statistics were expressed as mean \pm standard deviation (SD) and frequency.

\section{Results}

The mean age at the time of diagnosis was $3.9 \pm 4.6$ years (range, one months to 18 years) and $5.6 \pm 4.5$ (range, 6 months to 14.5 years) years at the time of transcatheter treatment (Table I). The mean follow-up period was 7.4 \pm 4.5 years (range, one to 17.5 years), while treated patients were followed for a mean duration of $8.3 \pm 4.4$ years (range, 2-15.5 years). Coronary artery fistulae were revealed during echocardiographic examination in 32 (57.1\%) patients with murmur, while $15(26.8 \%)$ patients were diagnosed previously with other CHD's, five $(8.9 \%)$ patients experienced cyanosis, and four $(7.1 \%)$ patients had chest pain. Although 24 patients $(42.9 \%)$ had associated lesions, interventionally or surgically-treated patients had no associated CHD's patients who were treated with either surgical or transcatheter techniques did not have an associated intracardiac defect. Ventricular septal defect, atrial septal defect and tetralogy of Fallot were common types of associated lesions (Table II). For 17 patients $(30.4 \%)$, the RCA was the most common site of origin for CAF. Meanwhile 11 patients $(19.6 \%)$ had CAF that originated at the LMCA, and 11 patients $(19.6 \%)$ had CAF originating at the LAD. Coronary artery fistulae originated from the circumflex coronary artery $(C x)$ in five patients $(8.9 \%)$, while one patient had CAF that originated in both the RCA and LAD. The termination site of fistulae was identified by catheter angiography. In 23 patients $(41.1 \%)$, the CAF terminated in the RV, while the left ventricle (LV) was the termination site in only one patient $(1.8 \%)$. Other termination 
Table I. Demographic characteristics of 15 patients treated with transcatheter and/or surgery.

\begin{tabular}{|c|c|c|c|c|c|c|c|}
\hline Diagnosis & $\begin{array}{l}\text { Treatment } \\
\text { age }\end{array}$ & Qp/Qs & Tract of & Treatment & Complication & Device & Outcome \\
\hline$\overline{\mathrm{CAF}}$ & 14.5 years & 1.3 & RCA-RV & Transcatheter & No & Coils & Survive \\
\hline CAF & 1 years & 1.8 & Cx-RV & Transcatheter & No & Vascular plug & Survive \\
\hline CAF & 10 years & 1 & RCA-LA & Transcatheter & No & Vascular plug & Survive \\
\hline CAF & 10 years & 1.4 & RCA-RA & Transcatheter & No & Vascular plug & Survive \\
\hline CAF & 4 years & 1.2 & RCA-RA & Transcatheter & No & Vascular plug & Survive \\
\hline CAF & 5.5 years & 1.6 & RCA-RV & Transcatheter & No & Vascular plug & Survive \\
\hline CAF & 5.5 years & 1.4 & LAD-RV & Transcatheter & No & Coils & Survive \\
\hline CAF & 3 years & 1.8 & RCA-RV & Transcatheter & $\begin{array}{l}\text { Coil } \\
\text { embolization }\end{array}$ & Coils & Survive \\
\hline CAF & 2 years & 1.4 & LMCA-RV & Transcatheter & $\begin{array}{l}\text { Myocardial } \\
\text { infarction }\end{array}$ & $\begin{array}{l}\text { Coils+Tissue } \\
\text { adhesive glue }\end{array}$ & Survive \\
\hline CAF & 6 months & 3.2 & RCA-RV & Transcatheter & No & Vascular plug & Survive \\
\hline CAF & 9 years & 1 & Cx-LA & $\begin{array}{l}\text { Surgical (unsuccessful } \\
\text { transcatheter) }\end{array}$ & No & - & Survive \\
\hline CAF & 6 months & 2.4 & RCA-RV & $\begin{array}{l}\text { Surgical (unsuccessful } \\
\text { transcatheter) }\end{array}$ & No & - & Survive \\
\hline CAF & 10 months & 1.6 & LMCA-RV & Surgical & RBBB & - & Survive \\
\hline CAF & 4 years & 1.7 & RCA-RA & Surgical & No & - & Survive \\
\hline CAF & 5 years & 1 & LMCA-LV & Surgical & No & - & Survive \\
\hline
\end{tabular}

CAF: coronary artery fistula, Cx: circumflex coronary artery, LA: left atrium, LAD: left anterior descending coronary artery, LMCA: left main coronary artery, LV: left ventricle, RA: right atrium, RBBB: right bundle branch block, RCA: right coronary artery, $\mathrm{RV}$ : right ventricle, Qp/Qs ratio: pulmonary to systemic blood flow ratio.

Table II. Additional congenital heart diseases associated with coronary artery fistula.

\begin{tabular}{lll}
\hline Congenital heart disease & N & $\%$ \\
\hline TOF & 4 & 7.1 \\
VSD, ASD, PS & 3 & 5.4 \\
VSD, DCRV & 3 & 5.4 \\
ASD & 3 & 5.4 \\
PDA & 2 & 3.6 \\
BAV, AS, CoA & 1 & 1.8 \\
DSM, DAA & 1 & 1.8 \\
BAV, AS & 1 & 1.8 \\
VSD, PDA & 1 & 1.8 \\
VSD, ASD & 1 & 1.8 \\
DCRV & 1 & 1.8 \\
VSD & 1 & 1.8 \\
TA & 1 & 1.8 \\
PS & 1 & 1.8 \\
\hline Total & 24 & 42.9
\end{tabular}

AS: aortic stenosis, ASD: atrial septal defect, BAV: bicuspid aortic valv, CHD: congenital heart disease, CoA: coarctation of the aorta, DAA: double aortic arch, DCRV: double chamber right ventricle, DSM: discrete subaortic membrane, PDA: patent ductus arteriosus, PS: pulmonary stenosis, TA: truncus arteriosus, TOF: tetralogy of Fallot, VSD: ventricular septal defect. 
sites were the pulmonary artery in 16 patients $(28.6 \%)$, the right atrium (RA) in three patients $(5.4 \%)$, and the left atrium (LA) in two patients $(3.6 \%)$ (Table I). Cardiac catheterisation wasn't performed in four patients because they had small and asymptomatic fistulae. These patients were followed regularly. Initially small fistulae were evaluated by cardiac catheterisation. However, small fistulae couldn't be evaluated by catheterisation in seven $(12.5 \%)$ patients because selective coronary angiography was not performed. Cardiac catheterisation was not performed in the last four patients with small and asymptomatic fistulae, and instead they were followed periodically using echocardiography. Small fistulae grew over time in three patients; two of these patients were treated with transcatheter occlusion while one underwent surgery (12.5 years, 5 years and 4.5 years after receiving their diagnoses respectively). Fifteen patients $(26.8 \%)$ were treated by either transcatheter or surgical means. Forty-one patients (73.2\%) needed no treatment, since their fistulae were small,

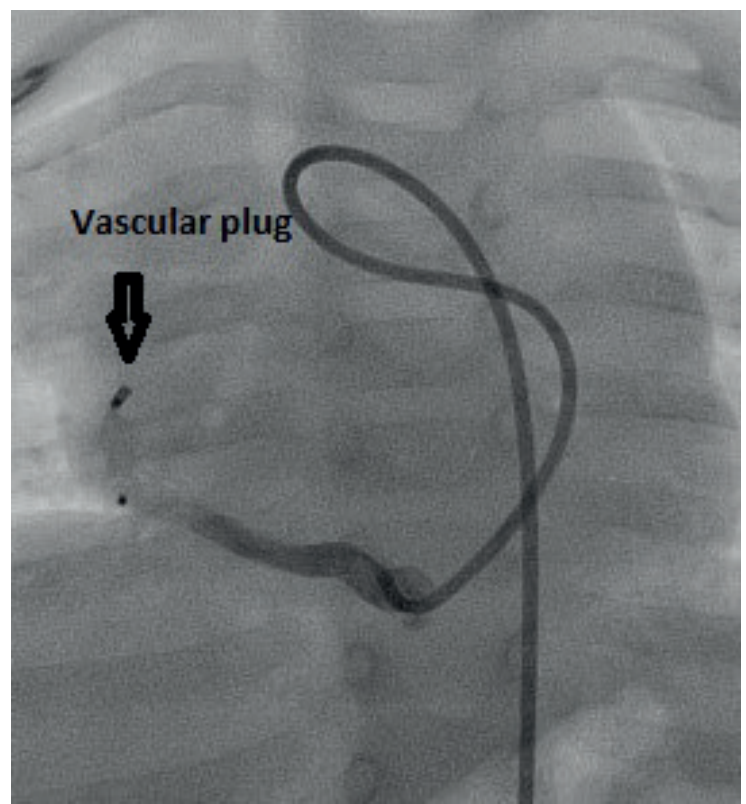

Fig. 1. A $6 \times 7 \mathrm{~mm}$ vascular plug (Cera ${ }^{\mathrm{TM}}$ Lifetech Scientific Corporation, China) placed in the distal part of the dilated circumferential coronary artery of a one year old child is seen. No residual shunt was observed in the control angiogram. asymptomatic, and had low shunting. The mean pulmonary to systemic blood flow (Qp) Qs) ratio among treated patients was 1.6 (min: 1, max: 3.2). The most common treatment indication was coronary artery dilation, which was found in $28.6 \%$ of patients, followed by coronary steal syndrome in $16 \%$ of patients. Other indications included the enlargement of cardiac chambers among $14.3 \%$ of patients, a Qp/Qs ratio greater than 1.5 in $12.5 \%$ of patients, and the presence of congestive heart failure symptoms in $9 \%$ of patients. A transcatheter approach was preferred to treat CAF in ten patients. Transcatheter treatment failed in two patients who had tortious and narrow fistulae because the device could not be placed distal to the fistulae. Instead, surgical correction was performed in these patients.

Coronary artery fistulae were occluded interventionally in six patients using vascular plugs (Fig. 1) (Cera $^{\mathrm{TM}}$ Lifetech Scientific

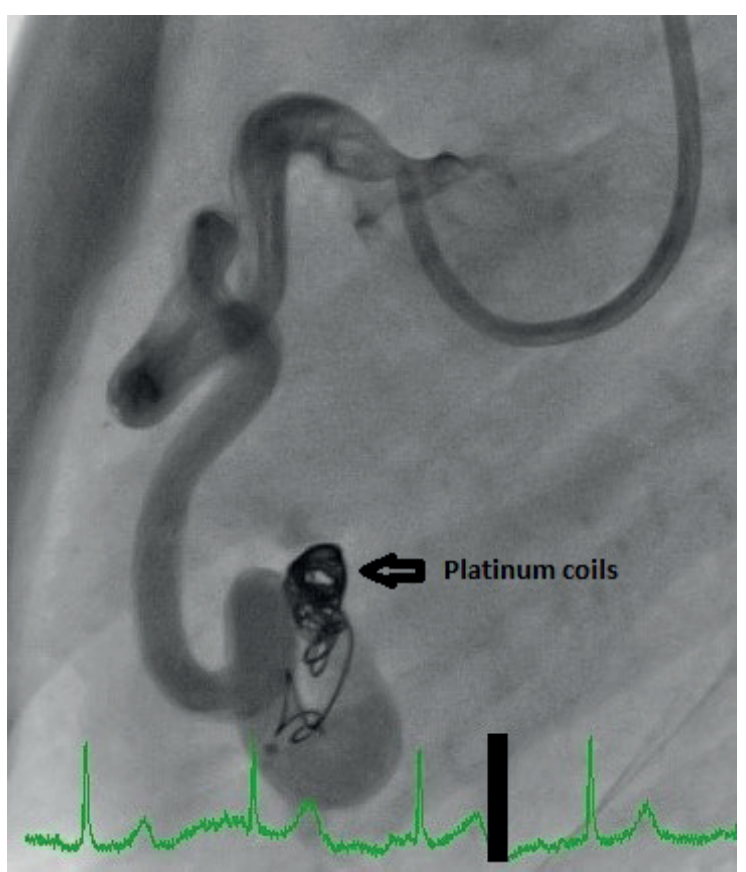

Fig. 2. In a 14.5 year-old patient, five platinum coils placed in the right coronary artery from the site where the coronary artery is dilated and curved and the distal part drained in the right ventricle is seen. No residual shunt was observed in the control angiogram. 
Corporation, China), (Amplatzer ${ }^{\mathrm{TM}}$ Vascular Plug, St. Jude Medical, USA). Platinum coils (Boston Scientific. USA) were used to occlude the CAF in three patients (Fig. 2), while the platinum coil plus tissue adhesive glue was used in one patient. Five patients were successfully treated with surgery, and transcatheter treatment attempted in two patients before surgery A large tunnel-like structure between the LCA and RV in one patient, between the RCA and $\mathrm{RV}$ in one patient, and between the RCA and RA in other two patients were observed. Direct surgical corrections were performed in these four patients. Different techniques for surgical closure of coronary fistulae have been used in this subset of patients. In a patient with favourable morphology, the fistula could be closed using simple ligation. However, it should be noted that this approach was not recommended. In four patients, fistulae were closed using cardiopulmonary bypass and cardioplegic arrest. In patients with coronary to RA fistulae, the communication was visualised after the onset of cardiopulmonary bypass but before cardioplegic arrest. This sequence was helpful for identifying the right atrial opening of the fistula. In another patient with a RCA-RV fistula and aneurysmal dilatation of the RCA, the orifice was sutured and closed primarily through the RCA, with identification of distal continuity and the orifice of the acute margin branch. Transesophageal echocardiography was used to confirm complete closure of the fistulae in most recent cases.

Early complications developed in two patients during the transcatheter procedure. At the beginning of the procedure, a $4 \mathrm{~mm} \times 3 \mathrm{~cm}$ coil was embolized into the right ventricle and then into the left pulmonary artery. The procedure was continued by deploying a coil that was one size larger. Closure was successfully completed with using four coils. To remove the embolized coil which is in the left pulmonary artery, $5 \mathrm{~F}$ sheat was replaced with $7 F$ long sheat. With the help of microsnare catheter, the embolized coil was caught in the left pulmonary artery and was safely removed by taking into the long sheat. To close the CAF, five platinum coils were used in our second patient who has developed an early complication. The subsequent angiogram showed that the fistula was not completely closed and there was a significant amount of transition of contrast material. A flow-guided microcatheter (1.2 Fr Marathon catheter, Covidien, Mansfield, Massachusetts, USA) was navigated using a micro-guidewire (Mirage 8, Covidien) into the feeding artery as close as possible to the fistula. The microcatheter was washed with $5 \%$ dextrose. A 50\% mixture of NBCA and Lipiodol (Guerbet LLC., Bloomington, Indiana, USA) was injected under digital subtraction angiography control. After correct penetration of the glue, the microcatheter was rapidly withdrawn. During this procedure, the electrocardiogram showed significant ST segment elevation. A control angiography was performed considering there may be myocardial infarction due to the reflux of the tissue adhesion material. The angiography demonstrated some degree of occlusion in the LAD and Cx branches with LAD edge irregularities, but the lumen of LAD and Cx were patent. Seven days after the procedure, coronary angiography was repeated and normal coronary flow patterns in the LAD and Cx were documented with previously-occluded branches and the persistence of LAD edge irregularities. Ejection and shortening fraction were measured using echocardiography as $52 \%$ and $24 \%$, respectively, and echocardiography showed abnormalities in the apical and septal wall motion. This patient was diagnosed with myocardial infarction. He received streptokinase for three days, heparin for four days, and then acetylsalicylic acid $(5 \mathrm{mg} / \mathrm{kg} /$ day) treatment for six months. During the last visit, echocardiographic examination showed normal cardiac function. Right bundle branch block (RBBB) was seen as an early surgical complication in one patient. RBBB due to surgical trauma improved spontaneously at the last follow-up. There were no late complications identified in treated patients. Medical records showed that 51 (91.1\%) patients were followed regularly, while five patients $(8.9 \%)$ were 
lost during follow-up. No treatment-related deaths were observed. At the time of discharge, anticoagulant therapy was administered to only one patient who had a myocardial infarction. No residual shunting or recanalization was observed among treated patients in long-term follow-up.

\section{Discussion}

Coronary artery fistulae are usually congenital in nature, and represent approximately $0.2-0.4 \%$ of all congenital heart diseases. ${ }^{3,4,9}$ It is reported that CAF commonly originates from the RCA, LAD or Cx and terminates in the right ventricle, right atrium and pulmonary artery. ${ }^{1-4,9-11}$ The origin and termination sites for CAF in our patients were similar to those reported in the literature. Fistula-related complications tend to increase with age. Complication rates were reported to be $11 \%$ in patients younger than 20 years old, while these rates increased to $35 \%$ in patients older than 20 years old. ${ }^{12,13}$

Enlargement of the cardiac chamber, left ventricle hypertrophy, congestive heart failure, coronary artery dilation, dysrhythmia, angina pectoris, chronic myocardial ischaemia that causes myocardial dysfunction, papillary muscle dysfunction, valve regurgitation and cardiomyopathy are common complications reported in patients with CAF. ${ }^{2-5,8}$ Our results were comparable to the literature, which showed that coronary artery dilation, coronary steal syndrome, enlargement in the heart chamber, and congestive heart failure were common complications that developed in our patients.

In the literature, associated lesions have been reported to be found in $20-60 \%$ of patients. ${ }^{1-4,9}$ We described CHD in $42.9 \%$ of patients, which was in agreement with previous findings in the literature.

Echocardiography is a highly-useful, noninvasive imaging modality that can determine the extent of dilation in the heart chamber, the origin, size, and drainage sites of fistulae. . $-5,5,10,14^{-14}$
Angiography provides detailed information about fistulae such as size, course, origin and drainage sites, and it also may help illustrate stenosis and associated CHD., ${ }^{3,15}$ Angiography may also be used in therapeutic intervention.,15 In addition to echocardiography and catheters, angiography was the main diagnostic tool used in our study. We also effectively employed transesophageal echocardiography to determine the origin site and the termination chamber of fistulae during surgical intervention.

In this study, CAF could not be confirmed in seven patients $(12.5 \%)$ who were suspected to have fistulae based on echocardiographic images, because selective coronary angiography was not performed. It is difficult to predict how small fistulae will change over time; some may enlarge over time in some patients, while they may narrow and close spontaneously in others. In this study, CAF became larger over time among three patients; two of these CAF were occluded interventionally, while the other was closed surgically. We suggest that small fistulae do not require cardiac catheterisation if they are differentiated from abnormal origin of the coronary artery. However, they should be examined periodically by echocardiography because it is probable that they may enlarge and become symptomatic during the follow-up period.

It is well known that small CAF's can grow with age and may cause severe haemodynamic, thrombotic, or ischaemic complications during adulthood. Some authors reported that treatment of CAF in adulthood seems to be more difficult due to the increased tortuosity of the CAF. ${ }^{16}$ Although it is controversial to treat asymptomatic patients with a small fistula, it is recommended to treat patients who have symptoms or large fistulae since complications may be observed frequently in these patients. ${ }^{1-4,9}$ Since complications and late symptoms are likely in patients with significant shunting (Qp/Qs ratio>1.5), these patients should be treated soon after their diagnosis. ${ }^{2,4,9,17}$ Improvement in fistula-related dilation of the coronary artery was reported in mid-long 
term after treatment. ${ }^{5}$ Early intervention is also important for myoproliferation, which helps restore normal calibration in the aneurysmal coronary segment. Many studies indicate that transcatheter intervention has a high success rate with less complications compared to surgery. ${ }^{1-4,18-21}$ The major advantage of transcatheter closure is that the procedure is performed without cardiopulmonary bypass and median sternotomy, which prevents iatrogenic complications. ${ }^{1-4}$ A shorter recovery time, reduced mortality rates, and lower cost are other advantages of this treatment. However, Collins et al. ${ }^{22}$ showed that vessel tortuosity and the calibre of the lumen seem to limit delivery of the device.

A surgical approach for closure of CAF may be performed safely regardless of the patient's age or the size of the fistula. ${ }^{23}$ Surgical closure is the treatment of choice when CAF is associated with congenital heart defects that also require surgery. Surgery offers an easier way to correct large aneurysms and proximal fistulae. The epicardial and endocardial approaches are two techniques that are used in surgical closure. The life expectancy of CAF patients is normal, with 9-19\% recurrence after transcatheter treatment and $25 \%$ after surgical ligation. ${ }^{10}$ The recurrence rate in published series varies significantly, from no incidence of recanalization in some studies, to $10-15 \%$ rate of reoccurrence in others. ${ }^{1,24,25}$ Jama et al..$^{20}$ reported $30 \%$ of patients had residual shunting, but less than $1 \%$ of these patients needed reintervention. Transcatheter occlusion was the preferred method for treating CAF in our institution. Although the balloon occlusion test prolongs the procedure, it is valuable for identifying out the most distal functional branch of the CAF, but it cannot always be performed. In our clinic, fistulae were closed using the most distal part to prevent disrupting myocardial perfusion. There were no instances of thrombotic complications during the period of involution among the transcatheter-treated patients group, so none of the patients needed antithrombotic therapy. Surgery was another treatment option when transcatheter intervention failed, or when transcatheter device closure was unsuitable. Our success rate for transcatheter treatment of CAF was $83.3 \%$.

Although anticoagulant recommendations are not available, anticoagulation after transcatheter closure is recommended in some studies. ${ }^{1,26}$ In this study, anticoagulant agents were administered to one patient who experienced a myocardial infarction caused by tissue adhesive. However, the remainder of patients did not need anticoagulation therapy. We do not recommend routine anticoagulant therapy after a transcatheter procedure unless there is a certain indication.

The mean follow-up period of the treated group was $8.3 \pm 4.4$ years, and it was impressive that no patients had residual shunting or recanalization during this time. Late complications, such as thrombus formation and myocardial ischaemia, were not seen in this group. It is recommended that all patients with closed CAF be followed regularly, since residual shunting and recanalization continue to be potential dangers for them in the long-term.

The risk of complications related to transcatheter or surgical approaches is low. Dysrhythmia, changes in the ST-T segment, myocardial infarction, coronary artery spasm, fistula dissection and coil embolisation are some of the complications that may develop after treatment. ${ }^{1,25,13,20,27}$ The mortality rate among patients with complications was reported to be $0-2 \% .^{13,27}$ We reported two complications during the transcatheter procedure. One of them was coil embolisation, while another was myocardial infarction in the patient that received an injection of tissue adhesive. Based on this experience, we determined that tissue adhesive could translocated to and block the coronary artery, which may cause myocardial infarction. RBBB developed in one patient due to surgery and this improved spontaneously at the last follow-up. There was no procedurerelated death, and no reinterventions required for residual shunting or recanalization in this study. 
A retrospective study is a significant disadvantage in the collection and evaluation of data. The change of medical equipment according to technological developments during this period is a major limitation. Transcatheter closure of the fistulae is considered a limitation among a small number of patients. Considering we had data from 56 patients, this may be a factor to consider.

In conclusion, echocardiography is an adequate tool for monitoring small, asymptomatic and hemodynamically-insignificant fistulae. Large, symptomatic fistulae and fistulae that cause haemodynamic disturbance and dilation of the coronary artery should be treated. Transcatheter closure should be the treatment of choice for CAF since it offers a high success rate and a low rate of complications with a shortened hospital stay. In patients with fistulas that are not appropriate for transcatheter occlusion, surgery is an effective alternative.

\section{REFERENCES}

1. Christmann M, Hoop R, Dave H, Quandt D, Knirsch W, Kretschmar O. Closure of coronary artery fistula in childhood: treatment techniques and long-term follow-up. Clin Res Cardiol 2017; 106: 211-218.

2. Buccheri D, Chirco PR, Geraci S, Caramanno G, Cortese B. Coronary artery fistulae: anatomy, diagnosis and management strategies. Heart Lung Circ 2018; 27: 940-951.

3. Holzer R, Johnson R, Ciotti G, Pozzi M, Kitchiner D. Review of an institutional experience of coronary arterial fistulas in childhood set in context of review of the literature. Cardiol Young 2004; 14: 380-385.

4. Lo MH, Lin IC, Hsieh KS, et al. Mid- to long-term follow-up of pediatric patients with coronary artery fistula. J Formos Med Assoc 2016; 115: 571-576.

5. Ponthier L, Brenot P, Lambert V, Petit J, Riou JY, Baruteau AE. Closure of isolated congenital coronary artery fistula: long-term outcomes and rate of reintervention. Pediatr Cardiol 2015; 36: 1728-1734.

6. Chandra N, Sarkar A, Pande A. Large congenital coronary arteriovenous fistula between the left main coronary artery and right superior vena cava, associated with aneurysmal dilatation of the left main coronary artery: rare case report. Cardiol Young 2015; 25: 143-145.
7. Jariwala U, Hasan RK, Thorn EM, Zakaria S. An unusual case of infective endocarditis involving a right coronary artery to superior vena cava fistula. Catheter Cardiovasc Interv 2015; 85: 620-624.

8. Doksöz Ö, Küçük M, Güven B, et al. Congenital coronary arteriovenous fistulas: an evaluation of 10 pediatric patients. J Thorac Cardiovasc Surg 2015; 23: $217-221$

9. Chiu CZ, Shyu KG, Cheng JJ, et al. Angiographic and clinical manifestations of coronary fistulas in Chinese people: 15-year experience. Circ J 2008; 72 : 1242-1248.

10. Challoumas D, Pericleous A, Dimitrakaki IA, Danelatos C, Dimitrakakis G. Coronary arteriovenous fistulae: a review. Int J Angiol 2014; 23: $1-10$.

11. Kiefer TL, Crowley AL, Jaggers J, Harrison JK Coronary arteriovenous fistulae: the complexity of coronary artery-to-coronary sinus connections. Tex Heart Inst J 2012; 39: 218-222.

12. Carrel T, Tkebuchava T, Jenni R, Arbenz U, Turina $\mathrm{M}$. Congenital coronary fistulas in children and adults: diagnosis, surgical technique and results. Cardiology 1996; 87: 325-330.

13. Armsby LR, Keane JF, Sherwood MC, Forbess JM, Perry SB, Lock JE. Management of coronary artery fistulae. Patient selection and results of transcatheter closure. J Am Coll Cardiol 2002; 39: 1026-1032.

14. Dawn B, Talley JD, Prince CR, et al. Two-dimensional and Doppler transesophageal echocardiographic delineation and flow characterization of anomalous coronary arteries in adults. J Am Soc Echocardiogr 2003; 16: 1274-1286.

15. Krishnamoorthy KM, Rao S. Transesophageal echocardiography for the diagnosis of coronary arteriovenous fistula. Int J Cardiol 2004; 96: 281-283.

16. Wang SS, Zhang ZW, Qian MY, Zhuang J, Zeng GH. Transcatheter closure of coronary arterial fistula in children and adolescents. Pediatr Int 2014; 56: 173179 .

17. Mitropoulos F, Samanidis G, Kalogris P, Michalis A. Tortuous right coronary artery to coronary sinus fistula. Interact Cardiovasc Thorac Surg 2011: 13: 672-674.

18. Trehan V, Yusuf J, Mukhopadhyay S, et al Transcatheter closure of coronary artery fistulas. Indian Heart J 2004; 56: 132-139.

19. Harikrishnan S, Bimal F, Ajithkumar V, et al Percutaneous treatment of congenital coronary arteriovenous fistulas. J Interv Cardiol 2011; 24: 208215. 
20. Jama A, Barsoum M, Bjarnason H, Holmes DR Jr, Rihal CS. Percutaneous closure of congenital coronary artery fistulae: results and angiographic follow up. JACC Cardiovasc Interv 2011; 4: 814-821.

21. Zhu XY, Zhang DZ, Han XM, et al. Transcatheter closure of congenital coronary artery fistulae: immediate and long-term follow-up results. Clin Cardiol 2009; 32: 506-512.

22. Collins N, Mehta R, Benson L, Horlick E. Percutaneous coronary artery fistula closure in adults: technical and procedural aspects. Catheter Cardiovasc Interv 2007; 69: 872-880.

23. Dodge-Khatami A, Mavroudis C, Backer CL. Congenital heart surgery nomenclature and database project: anomalies of the coronary arteries. Ann Thorac Surg 2000; 69: 270-297.
24. Abdelmoneim SS, Mookadam F, Moustafa S, et al. Coronary artery fistula: single-center experience spanning 17 years. J Interv Cardiol 2007; 20: 265-274.

25. Cheung DL, Au WK, Cheung HH, Chiu CS, Lee WT. Coronary artery fistulas: long-term results of surgical correction. Ann Thorac Surg 2001; 71: 190195.

26. Gowda ST, Latson LA, Kutty S, Prieto LR. Intermediate to long-term outcome following congenital coronary artery fistulae closure with focus on thrombus formation. Am J Cardiol 2011; 107: 302-308.

27. Qureshi SA, Tynan M. Catheter closure of coronary artery fistulas. J Interv Cardiol 2001; 14: 299-307. 